\title{
Field Investigation for Deck Slab Deterioration of In-Service Bridges in Korea
}

\author{
WooSeok Kim, Chan Jeoung', Yoseok Jeong ${ }^{1}$, Ilkeun Lee ${ }^{2}$ \\ ${ }^{1}$ Chungnam National University \\ 99 Dakhak-ro, Yusung-gu, Daejeon, Republic of Korea \\ wooseok@cnu.ac.kr; tony0946@hanmail.net; yoseoksi@gmail.com \\ ${ }^{2}$ Expressway \& Transportation Research Institute \\ 208-96 Dongbudaero922-gil, Hwaseong, Gyeonggi-do, Republic of Korea \\ lik@ex.co.kr
}

\section{Extended Abstract}

This study intends to investigate deck slabs of in-service bridges in Korea and determine most influencial parameters of exposed environments. Bridge deck slabs are crucial structural members directly supporting traffic loads and thus deteriorate earlier than other structural members. Thus, frequent maintenance plan is required for bridge deck slab and in fact many agencies spend most of their maintenance budget to repair and rehabilitate the bridge deck slabs.

In this study, 747 bridges were randomly selected to represent cold and moderate temperature region. The selected bridges have 6 to 44 years of service age with an average and median of 17.4 and 14.0 years, respectively. To investigate the damage of bridge deck slab, GPR (Ground Penetration Radar) system mounted on a vehicle and travelling at $80 \mathrm{~km} / \mathrm{h}$ was used. Deck slab damages were estimated through permittivity of deck slabs by radiating an electromagnetic pulse and measuring the rebounded pulse magnitude and timing.

Based on the collected GPR data, damage ratio was determined based on Korea Expressway Manual [1] and compared to eight key parameters: (1) annual average snowfall days; (2) annual average snowfall; (3) annual average number of freeze-thaw days; (4) annual average winter temperature; (5) altitude; (6) service age; (7) quantity of deicing chemical usage; and (8) equivalent traffic volume. Correlation for each parameter was determined to establish the influence of each parameter to deck slab deterioration and service age, number of freeze-thaw days and deicing chemical usage exhibited the highest correlations. Some studies reported traffic volume has a significant influence on bridge deck slab damage [2], but in this study traffic volume did not exhibit strong correlation. This was because the service ages of sampled bridges were young and traffic load tends to accelerate damages if initial damages exist [3]. To better predict of deck slab deterioration, mean and standard deviation was computed for collected data at each age, and was modelled using a normal distribution to represent uncertainties in deck slab deterioration. This study finally developed a deck slab deterioration chart along with service age. This chart can be used for bridge maintenance and budget planning.

\section{References}

[1] J. Seo and J. Lee, Manual of Bridge Deck's Deterioration Prevention. Seongnam, Republic of Korea: KEC, 2009.

[2] A. Nowak and M. Szerszen, "Life-Cycle Deterioration Model for Concrete Deck Slabs," in Proceedings of Life-Cycle Performance of Deteriorating Structures, ASCE, 2003, pp. 133-140.

[3] K. Dyduch, M. Szerszen, and J. F. Destrebecq, "Experimental investigation of fatigue strength of plain concrete under high compressive loading," Materials and Structures, vol. 27, pp. 505-509. 